Check for updates

Cite this: RSC Adv., 2018, 8, 42353

\title{
Fabrication of a polyvinylidene fluoride cactus-like nanofiber through one-step electrospinning $\dagger$
}

\begin{abstract}
Bilal Zaarour, (D) ab Lei Zhu, ${ }^{a}$ Chen Huang (D) a and Xiangyu Jin ${ }^{\text {*a }}$
Surface modification of fibers has attracted significant attention in different areas and applications. In this work, polyvinylidene fluoride (PVDF) cactus-like nanofibers were directly produced via electrospinning at a high relative humidity $(\mathrm{RH})$ of $62 \%$. The formation mechanism of the cactus structure was demonstrated. The effects of the $\mathrm{RH}$ on the fabrication of the cactus structure, crystalline phases, mechanical properties, hydrophobicity, and piezoelectric properties of the PVDF nanofibers were investigated. The results showed that the cactus-like nanofibers have a high crystallinity $\left(\Delta X_{c}\right)$, and an outstanding water contact angle (WCA), as well as good electrical outputs. We believe that the PVDF cactus structure can be used in many applications such as energy harvesting and self-cleaning surfaces.
\end{abstract}

Received 8th November 2018 Accepted 23rd November 2018

DOI: $10.1039 / c 8 \mathrm{ra09257e}$

rsc.li/rsc-advances excellent mechanical properties, ${ }^{18}$ a variety of morphological and structural properties, ${ }^{19}$ and their low density. ${ }^{20}$ Therefore, they have been used in different areas such as in superhydrophobic surfaces, ${ }^{21,22}$ energy harvesting, ${ }^{23}$ filtration, ${ }^{24,25}$ biomedical applications, ${ }^{26}$ catalysts, ${ }^{27}$ sensors, ${ }^{28}$ and so forth.

Nowadays, poly (vinylidene fluoride) (PVDF) is the polymer of choice for a variety of scientific researchers owing to its outstanding properties including its piezo-, pyro- and ferroelectricity, flexibility, thermal stability, high mechanical strength, chemical resistance, and its ability to be formed into different structures. ${ }^{17,29}$ Therefore, it has been increasingly used in different fields such as self-cleaning surfaces, ${ }^{30}$ energy harvesting, ${ }^{31}$ sensors,${ }^{32}$ filtration systems,${ }^{33}$ oil clean up materials ${ }^{34}$ and so on.

In the electrospinning process, a number of parameters including the relative humidity $(\mathrm{RH})$, temperature, polymer concentration, elasticity, electrical potential, and distance between the tip and the collector are known to affect the mechanical, physicochemical, and piezoelectric properties of the fibers, such as the fiber diameter, surface morphology, and the crystalline phases. ${ }^{35-42}$ However, the effects of the RH on the mechanical, physicochemical, and piezoelectric properties of the electrospun PVDF fibers have not been comprehensively studied so far.

Previously, we have reported the generation of cellulose acetate butyrate and polystyrene fibers with a grooved structure through electrospinning using a mixed solvent system containing a high boiling point solvent and a low boiling point solvent. ${ }^{3,43}$ Moreover, we have reported the fabrication of PVDF nanofibers by controlling the solvent systems and polymer concentration which have a maneuvering surface structure. ${ }^{19}$

In this work, we demonstrated the feasibility of fabricating PVDF cactus-like nanofibers at different levels of RH. Moreover, the relationship between the $\mathrm{RH}$ and the crystallinity $\left(\Delta X_{\mathrm{c}}\right)$,
${ }^{a}$ Engineering Research Center of Technical Textiles, Ministry of Education, College of Textiles, Donghua University, No. 2999 North Renmin Road, Songjiang, Shangha

stries Mechanical Engineering and Techniques Departme Mechanical and Electrical Engineering, Damascus University, Damascus, Syria

Electronic supplementary information (ESI) available: SEM pictures (lower magnification) of the PVDF fibers electrospun at different levels of $\mathrm{RH}$ are shown in Fig. S1. The diameter of the fiber is shown in Fig. S2. The current output generated by the PENGs based on the PVDF fiber webs at different levels of RH are shown in Fig. S3. See DOI: 10.1039/c8ra09257e 
mechanical properties, hydrophobicity, and piezoelectric properties of the PVDF nanofibers were investigated. To our knowledge, this is the first time that a cactus structure has been generated on the surface of electrospun PVDF nanofibers. We determined the mechanism of formation and explored the effect of the RH on the properties of the electrospun PVDF nanofibers. We concluded that the PVDF cactus structure has many advantages (e.g. high roughness, interior porosity, extraordinary water contact angle (WCA), and good electrical outputs), meaning it can be used in many different applications, in particular for energy harvesting and self-cleaning surfaces. This study could provide useful guidelines for the generation of cactus-like nanofibers via electrospinning.

\section{Experimental}

\subsection{Materials}

PVDF pellets $\left(M_{\mathrm{w}}=275000\right)$ were purchased from SigmaAldrich, USA. Acetone (ACE) and $N, N$-dimethylformamide (DMF) were purchased from Shanghai Chemical Reagents Co., Ltd, China. All chemicals were used without further purification.

\subsection{Methods}

Electrospinning: a 22\% (DMF/ACE) PVDF solution at a solvent ratio of $1: 8$ was prepared; the solution was loaded into a plastic syringe. The polymer concentration and the solvents used were selected based on their effects on the surface morphology and the spinnability of the fibers. ${ }^{19}$ In this work, the solvent ratio was the volume ratio, and the solution concentration was the weight/volume $(\mathrm{w} / \mathrm{v})\left(\mathrm{g} \mathrm{ml}^{-1}\right)$. A 21 gauge syringe needle was used as the spinneret, which was fixed onto a syringe pump (KDS 100, KD Scientific Inc., USA) connected to a high-voltage supplier (Tianjin Dongwen Co., Ltd., China). A grounded drum collector $(40 \mathrm{~cm}$ in length and $20 \mathrm{~cm}$ in diameter) was placed $18 \mathrm{~cm}$ away from the spinneret, and the rotating speed was set at $100 \mathrm{rpm}$. The setup for electrospinning is illustrated in Fig. 1a. All of the experiments were carried out at $20^{\circ} \mathrm{C}$ under different levels of relative humidity $(2 \%, 22 \%, 42 \%$, and $62 \%)$. The relative humidity was adjusted by the environmental humidity, which could be further fixed within a narrow window $( \pm 2 \%)$ by using a humidifier/dehumidifier. All of the samples were prepared at the feeding rate and applied voltage of $1.5 \mathrm{ml}$ $\mathrm{h}^{-1}$ and $18 \mathrm{kV}$, respectively. ${ }^{44}$

\subsection{Characterization}

The surface morphology and cross-section of the electrospun PVDF fibers were assessed using field emission scanning electron microscopy (FE-SEM, S-4800 Hitachi, Japan). Crosssections of the nanofibers were prepared by dipping them in liquid nitrogen for approximately $30 \mathrm{~s}$, and cutting them manually. The fiber diameter was measured using image processing software (ImageJ 1.45s). The relative humidity was checked using a Weather Thermometer Hygrometer (Anymeter TH101B, China). The thickness of the webs was adjusted at 100 $\mu \mathrm{m}$ using a micrometer (Anytime, USA). X-ray diffraction (XRD)

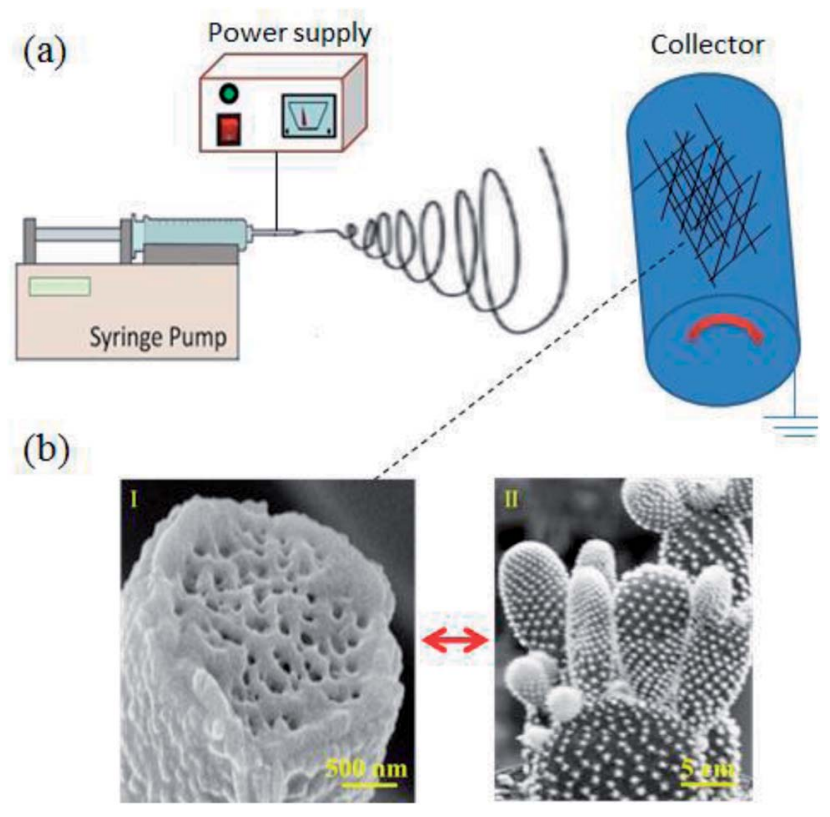

Fig. 1 (a) Schematic diagrams illustrating the electrospinning process. (b) Typical SEM image of the PVDF cactus-like fibers (I) and an optical image of a cactus leaf (II).

measurements were taken on a diffractometer (Panalytical XRD, Netherlands) using $\mathrm{Cu}$ radiation at $1.54 \AA$. The samples were scanned in the $2 \theta$ range of $5-30^{\circ}$. Fourier transform infrared (FTIR, USA) spectra were carried out on a Bruker Optics spectroscopy in the attenuated total reflection (ATR) mode. Differential scanning calorimetry (DSC, USA) was performed by heating the samples from 40 to $180{ }^{\circ} \mathrm{C}$ at the heating rate of $10^{\circ} \mathrm{C} \min ^{-1}$ in a nitrogen atmosphere. The mechanical properties of the PVDF fiber webs were tested using an Instron Universal Testing Machine (5967, USA) at a crosshead speed of $20 \mathrm{~mm} \mathrm{~min}^{-1}$. Six specimens with dimensions of $50 \times 15 \mathrm{~mm}^{2}$ were checked for each group. The static water contact angles (WCAs) were measured using the Dataphysics Contact Angle System (OCA, Germany) at the static sessile drop mode. The volume of the water droplets was $5 \mu$ l. For each sample, the results were obtained based on the WCA in eight different areas at room temperature. The piezoelectric nanogenerators (PENGs) were designed using the same process reported in our previous work. ${ }^{44}$ The open-circuit voltage and the short-circuit current of the PENGs with a working area of $15 \mathrm{~cm}^{2}$ were carried out via an oscilloscope (LeCroy, Wavesurfer 104MXs-B, USA) and current preamplifiers (Stanford Research SR570, USA), respectively, under an impact frequency of $5 \mathrm{~Hz}$, and a peak force of $10 \mathrm{~N}$.

\section{Results and discussion}

\subsection{Effect of the relative humidity on the formation of the PVDF cactus structure}

In this study, the PVDF nanofibers were fabricated at different levels of $\mathrm{RH}(2 \%, 22 \%, 42 \%$, and $62 \%)$, and the effect of the $\mathrm{RH}$ on their morphologies was studied. As shown in Fig. 2, smooth 

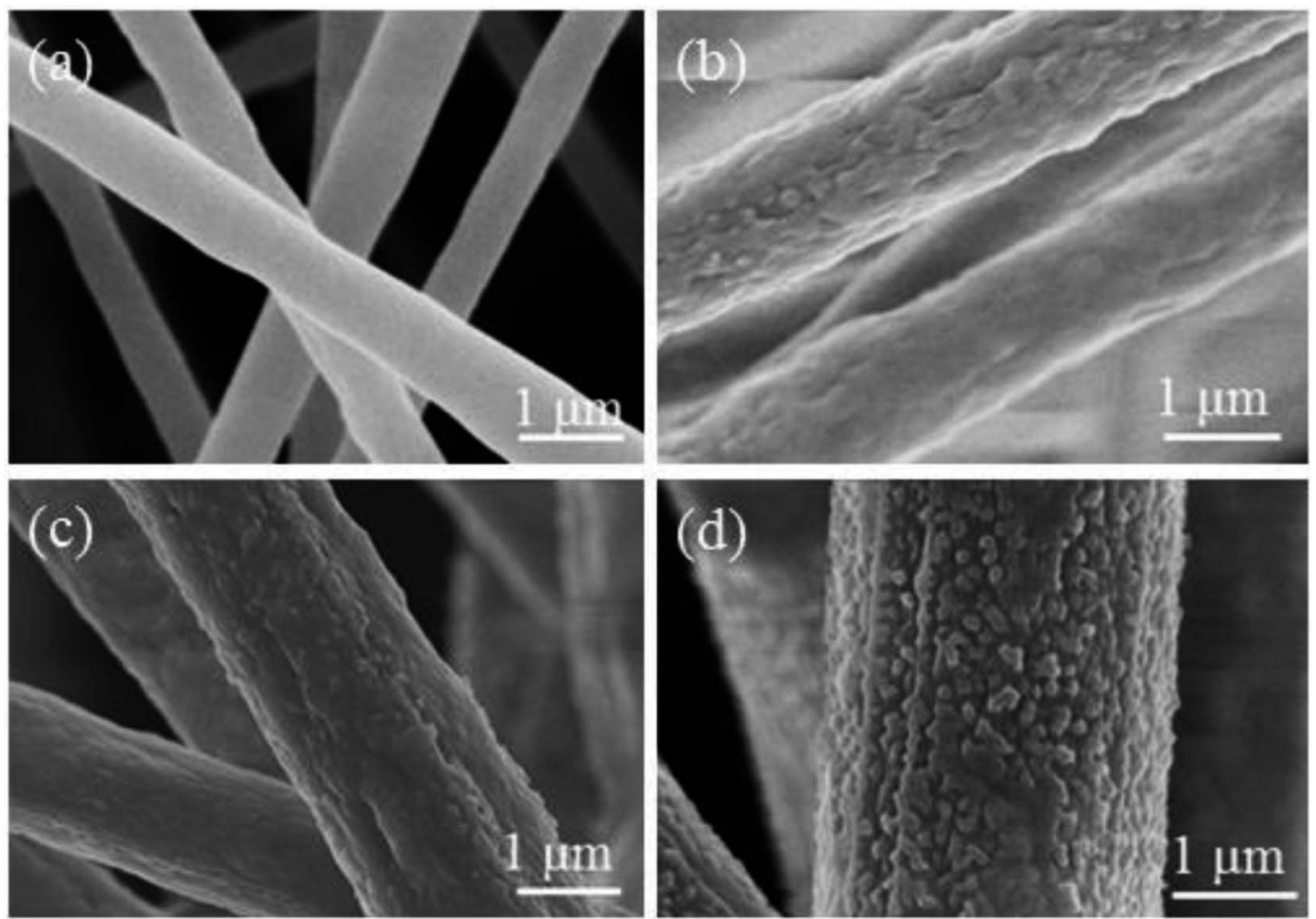

Fig. 2 SEM images of PVDF fibers electrospun at different levels of $\mathrm{RH}$ : (a) 2\%; (b) 22\%; (c) 42\%; and (d) $62 \%$.

fibers were formed at a RH of $2 \%$ (Fig. $2 \mathrm{a}$ and S1a $\uparrow$ ), whereas pillar fibers were produced at a RH of $22 \%$ and $42 \%$ (Fig. $2 \mathrm{~b}$, c, S1b and S1c $\dagger$ ), while cactus-like fibers were obtained at a RH of
62\% (Fig. 2d and S1d†). The average diameter of fibers formed increased from $836 \pm 99 \mathrm{~nm}$ at a $\mathrm{RH}$ of $2 \%$, to $2698 \pm 222 \mathrm{~nm}$ at a RH of $62 \%$ (Fig. S2 $\dagger$ ). In order to explore the mechanism of
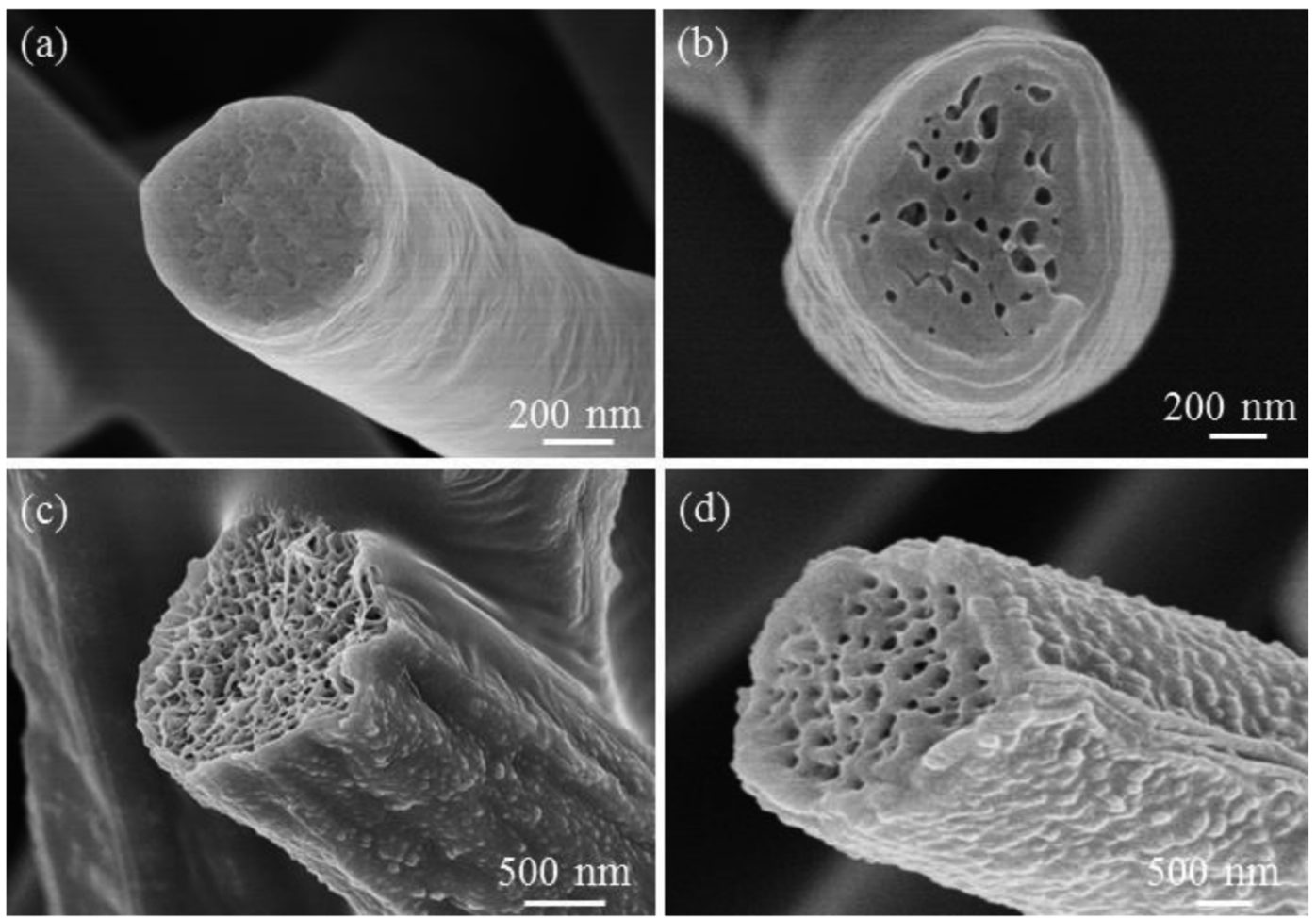

Fig. 3 Cross-sectional SEM images of PVDF fibers electrospun at different levels of RH: (a) 2\%, (b) 22\%, (c) 42\%, and (d) $62 \%$. 
(a)

(c) $\mathrm{RH}$.
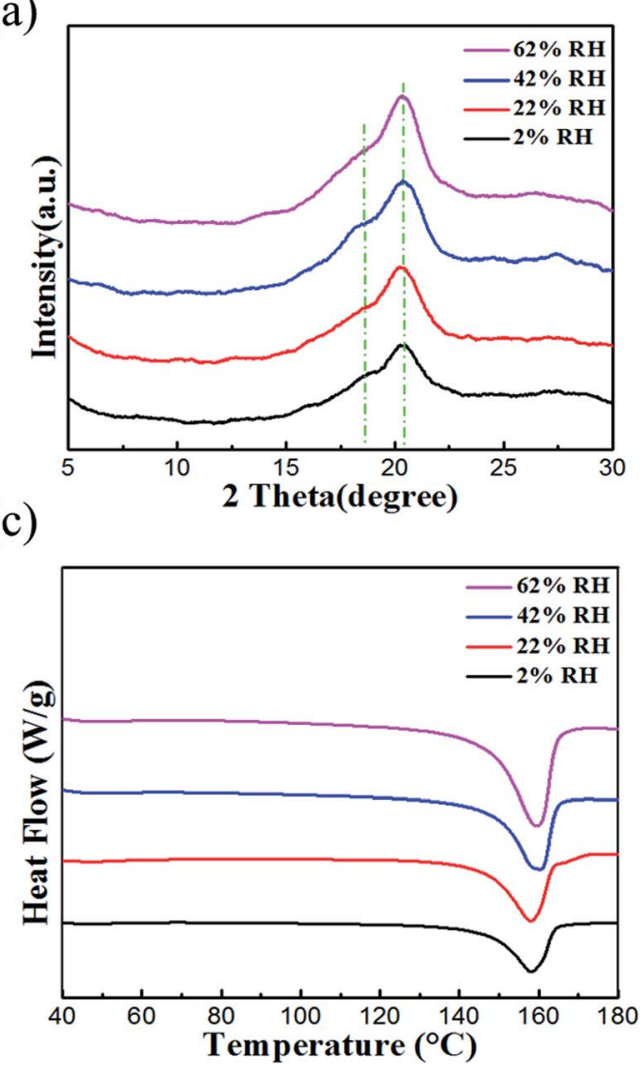

(b)

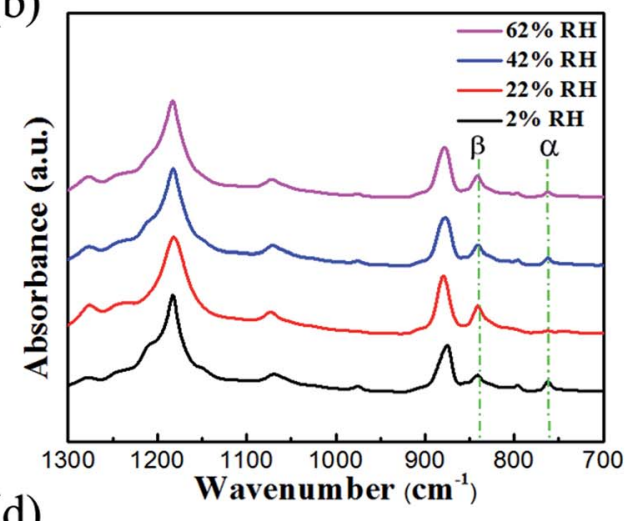

(d)

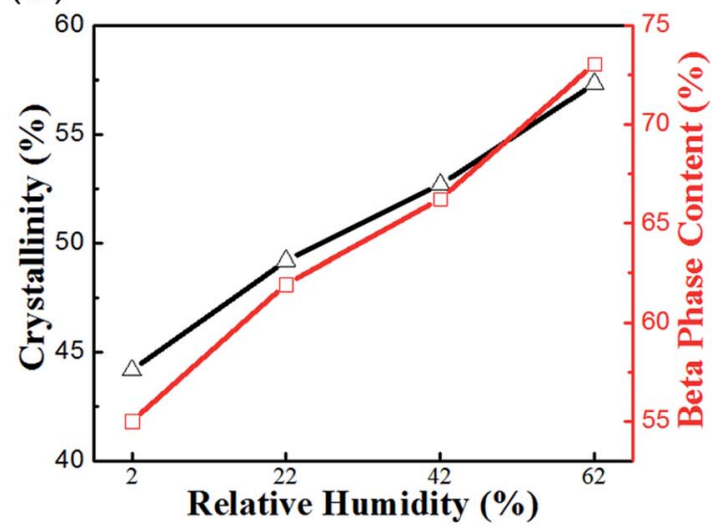

Fig. 4 (a) XRD patterns for the PVDF fiber webs electrospun at different levels of RH. (b) FTIR spectra for PVDF fiber webs electrospun at different levels of $\mathrm{RH}$. (c) DSC for PVDF fiber webs electrospun at different levels of $\mathrm{RH}$. (d) $\Delta X_{\mathrm{c}}$ and $F(\beta)$ for PVDF fiber webs prepared at different levels of

Table 1 The $\beta$ phase content and crystallinity of the PVDF fiber webs formed at different levels of $\mathrm{RH}$

\begin{tabular}{lllll}
\hline$F(\beta)$ & $\mathrm{RH}$ & & & \\
\cline { 2 - 5 } and $\Delta X_{\mathrm{c}}$ content & $2 \%$ & $22 \%$ & $42 \%$ & $62 \%$ \\
\hline$F(\beta)(\%)$ & 55 & 61.63 & 66.23 & 73.06 \\
$\Delta X \mathrm{c}(\%)$ & 44.17 & 49.2 & 52.7 & 57.32
\end{tabular}

formation for the cactus structure, a cross-section of the fibers formed at different levels of RH was analyzed. The solid interior was formed at a $\mathrm{RH}$ of $2 \%$ as there was no phase separation (Fig. 3a), while the interior pores were formed at a $\mathrm{RH}$ of $22 \%$, $42 \%$, and $62 \%$ (Fig. $3 \mathrm{~b}-\mathrm{d}$ ), and this can be ascribed to vapor induced phase separation (VIPS). ${ }^{\mathbf{1 9 , 4 5}}$ Herein, the mechanism of formation for the cactus structure can be attributed to this hypothesis. After combining the DMF/ACE solvents at a solvent ratio of $1: 8$, a mechanically strong sheath or layer was created first because of the rapid evaporation rate of ACE and the phase separation, which can resist the attack of the condensed water droplets. Furthermore, DMF (vapor pressure, $0.36 \mathrm{kPa}$ ) with a small portion of ACE (vapor pressure, $24 \mathrm{kPa}$ ) is trapped in the fiber; however ACE desperately wants to escape these constraints. The dense PVDF layer blocks most of the regions and inhibits the fast evaporation of ACE, but many weak points still exist, at which the evaporation rate of ACE is very high. Afterwards this erupting (DMF/ACE) PVDF solution is confronted by the surface water droplets and precipitates into the cactus structure.

\subsection{Effect of the RH on the crystalline phases of the PVDF nanofiber webs}

In order to determine the relationship between the $\mathrm{RH}$ and the crystalline phases of the PVDF nanofibers webs, the crystal structure of samples was checked at a RH of $2 \%, 22 \%, 42 \%$, and $62 \%$. The XRD patterns of the PVDF fibers electrospun at different levels of $\mathrm{RH}$ are shown in Fig. 4a. It has previously been reported that the $\alpha$ phase exhibits a peak at $2 \theta=18.4^{\circ}$, corresponding to the (020) crystal plane, while the sum $\beta$ phase showed a peak at $2 \theta=20.6^{\circ}$, corresponding to the (110) and (200) plane. ${ }^{46}$ The intensity of $\beta$ crystal phase increases by increasing the RH. To confirm the crystal phase structure, FTIR spectrophotometry was used. As shown in Fig. 4b, the characteristic bands of the $\beta$ phase crystals were observed at $840 \mathrm{~cm}^{-1}$ $\left(\mathrm{CH}_{2}\right.$ rocking) and $1274 \mathrm{~cm}^{-1}$ (trans band), while the $\alpha$ phase crystals were identified at bands 762 and $976 \mathrm{~cm}^{-1} \cdot{ }^{47,48}$ PVDF can have five polymorphs: the $\alpha$ and $\delta$ phase (TGTG') transgauche-trans-gauche, the $\beta$ phase (TTTT) all trans and (T3GT3G') 
(a)

.

for the $\gamma$ and $\varepsilon$ phases. ${ }^{49-52}$ Moreover, the $\beta$ phase content $[F(\beta)]$ of the PVDF fibers correlates to the piezoelectric response. ${ }^{53}$ Based on the eqn $\mathrm{S}(1)$, $\dagger$ the $F(\beta)$ content was $55 \%, 61.93 \%$, $66.23 \%$, and $73.06 \%$ at a $\mathrm{RH}$ of $2 \%, 22 \%, 42 \%$, and $62 \%$, respectively. The crystal degree of the samples $\left(\Delta X_{\mathrm{c}}\right)$ was measured using DSC analysis (Fig. 4c). The $\Delta X_{\mathrm{c}}$ content was calculated according to eqn $\mathrm{S}(2) \dagger$ and the $\Delta X_{\mathrm{c}}$ was $44.17 \%$, $49.2 \%, 52.7 \%$, and $57.32 \%$ at a RH of $2 \%, 22 \%, 42 \%$, and $62 \%$, respectively.

It should be noted that the $\Delta X_{\mathrm{c}}$ as well as the $F(\beta)$ increase when the $\mathrm{RH}$ increases owing to the enhanced degree of molecular orientation during the electrospinning of the PVDF fibers (Fig. $4 \mathrm{~d}$ ). In other words, $\Delta X_{\mathrm{c}}$ and $F(\beta)$ increase by increasing the evaporation time of the solvents. ${ }^{54}$ The $F(\beta)$ and $\Delta X_{\mathrm{c}}$ content of all of the fiber webs formed are listed in Table 1 .

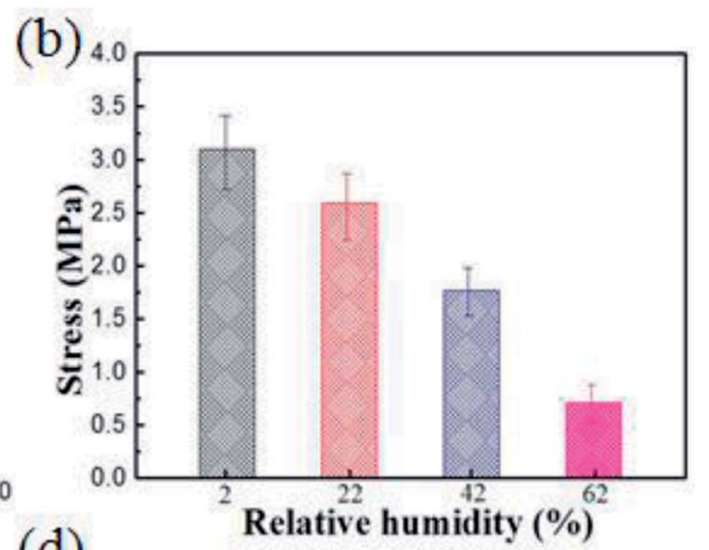

(d)

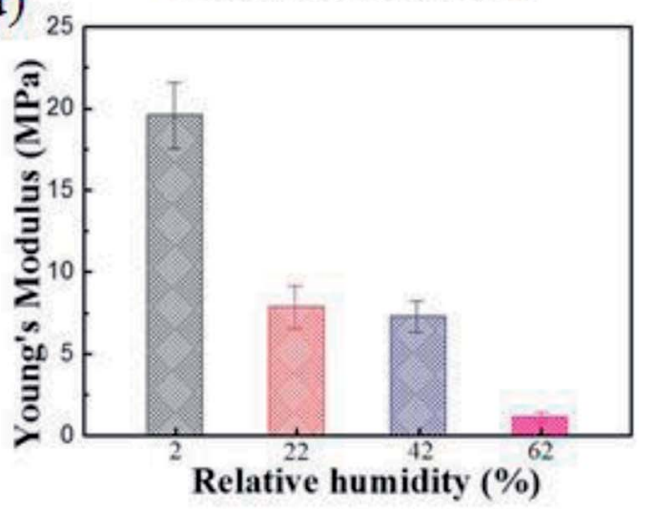

Fig. 5 A comparison of the tensile mechanical tests for the PVDF fiber webs electrospun at different levels of RH including: (a) stress-strain behavior; (b) stress; (c) strain; and (d) Young's modulus.

\subsection{Effect of the RH on the mechanical properties of the PVDF nanofiber webs}

The mechanical properties often measured, such as stress, strain, and Young's modulus, are dependent on both the strength of the individual fibers and the interactions between them. The results below illustrate how the mechanical properties of the PVDF fiber webs are affected by the $\mathrm{RH}$ during electrospinning.

Representative stress-strain curves for the PVDF fiber webs electrospun over a range of different relative humidities (2$62 \%$ ) are shown in Fig. 5a. The average stress, strain, and Young's modulus as a function of the RH are shown in Fig. 5bd, respectively. The results show that the PVDF fiber webs were the strongest at $2 \% \mathrm{RH}$, in which they had the highest stress (3.1 $\pm 0.38 \mathrm{MPa})$ and Young's modulus $(19.6 \pm 2.01 \mathrm{MPa})$. At a RH of $62 \%$, they exhibited the lowest stress $(0.713 \pm 0.13 \mathrm{MPa})$ and

Table 2 The mechanical properties of the PVDF fiber webs formed at different levels of RH

\begin{tabular}{|c|c|c|c|c|}
\hline \multirow{2}{*}{$\begin{array}{l}\text { Mechanical } \\
\text { properties }\end{array}$} & \multicolumn{4}{|l|}{$\mathrm{RH}$} \\
\hline & $2 \%$ & $22 \%$ & $42 \%$ & $62 \%$ \\
\hline Stress (MPa) & $3.1 \pm 0.38$ & $2.59 \pm 0.36$ & $1.77 \pm 0.22$ & $0.713 \pm 0.13$ \\
\hline Strain (\%) & $57.1 \pm 6.34$ & $133.6 \pm 15.9$ & $113 \pm 11.3$ & $58.6 \pm 6.11$ \\
\hline Young's modulus & $19.6 \pm 2.01$ & $7.9 \pm 1.02$ & $7.3 \pm 0.91$ & $1.2 \pm 0.18$ \\
\hline
\end{tabular}

(MPa) 


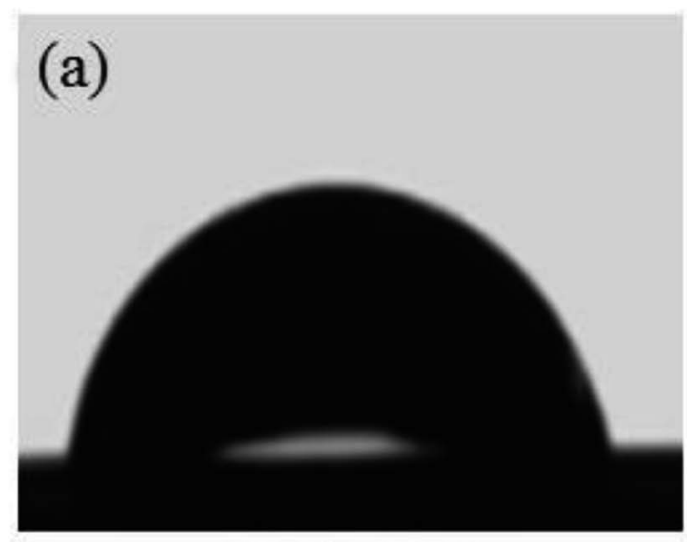

$81 \pm 4.6^{\circ}$

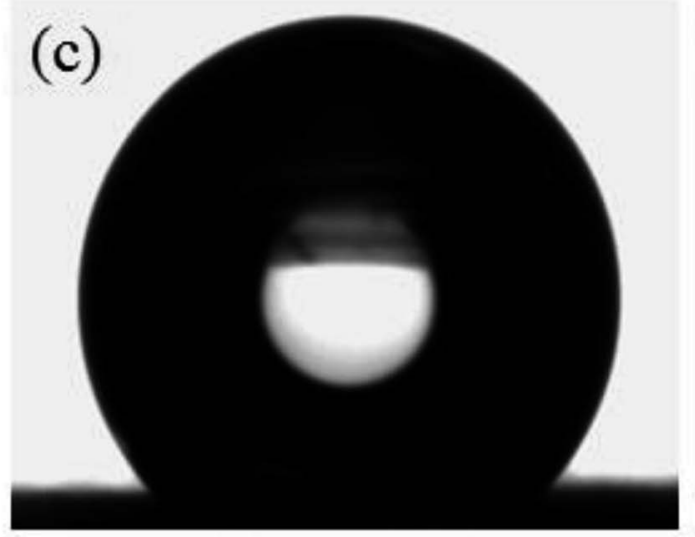

$135 \pm 3.2^{\circ}$

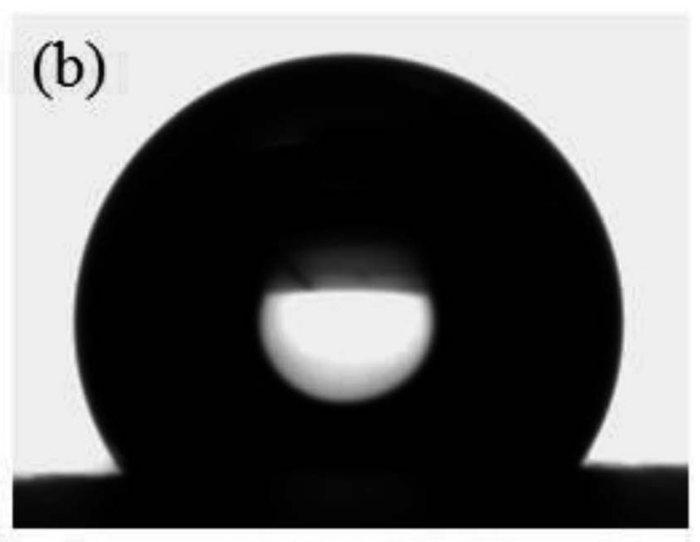

$128 \pm 3.8^{\circ}$

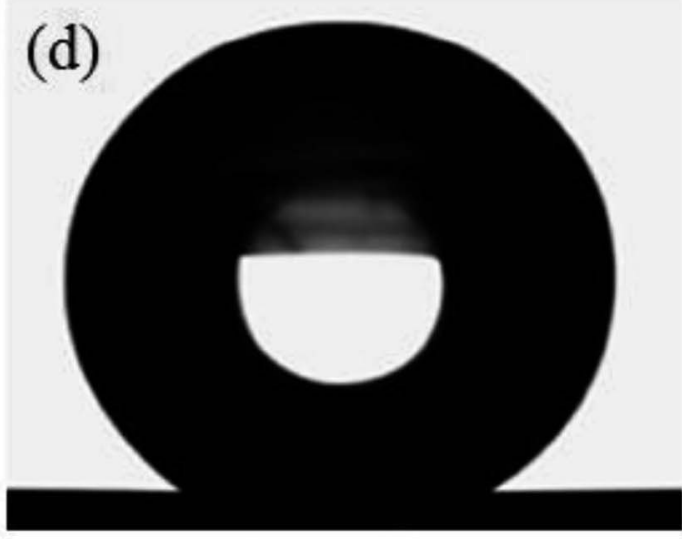

$156 \pm 2.5^{\circ}$

Fig. 6 Representative images of the water droplets and the WCA on the surface of the PVDF fiber webs electrospun at different levels of RH: (a) $2 \%$; (b) $22 \%$; (c) $42 \%$; and (d) $62 \%$.

Young's modulus $(1.2 \pm 0.18 \mathrm{MPa})$. Both the stress and the Young's modulus of the webs decreased when the RH was increased. The results indicate that the RH plays an important role in determining the mechanical properties of the PVDF nanofiber webs.

Generally, there is a negative linear relationship between the mechanical properties of the PVDF nanofiber webs and the RH. This could be visually noticed after the spinning was completed.

At a low RH, the fiber webs can be handled by hand and appeared uniform and well integrated, whereas the webs had a fluffy, cotton-like texture at a high RH owing to poor fiber- fiber bonding. The reason for this poor bonding at a high RH could be ascribed to the phase separation stimulated by the presence of water. A glassy skin forms soon after the polymer jet comes into contact with the surrounding air. As the glassy skin is created, the amount of residual solvent on it is reduced. Consequently, the adhesion between each fiber-fiber will be fragile as the residual solvent is essential to soldering the fiber junctions together. ${ }^{35}$ The mechanical properties of all of the fiber webs obtained at different levels of $\mathrm{RH}$ are summarized in Table 2.

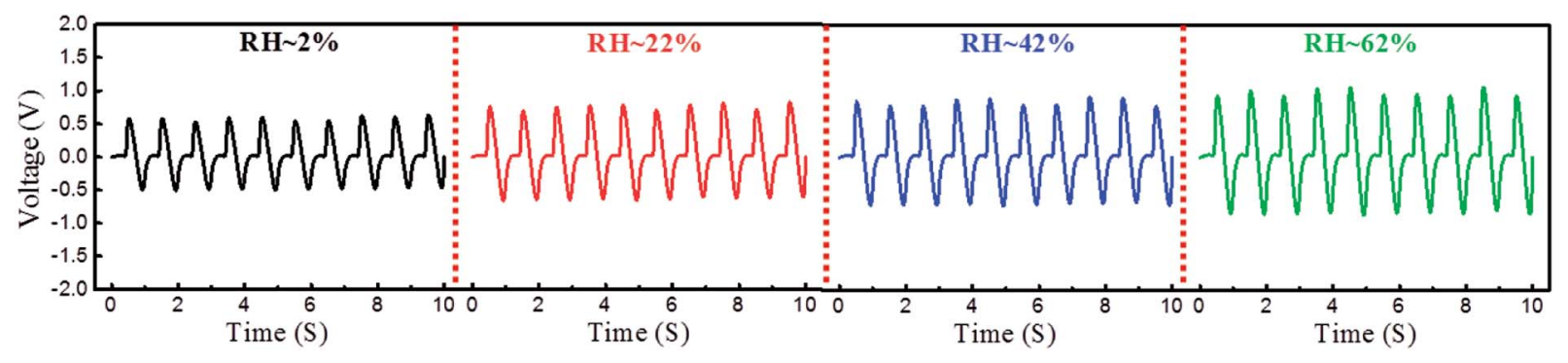

Fig. 7 Voltage output generated by the PENGs based on the PVDF fiber webs at different levels of RH. 


\subsection{Effect of the RH on the hydrophobicity of the PVDF} nanofiber webs

The WCAs of the electrospun PVDF nanofibers were examined to investigate the hydrophobicity of the webs. Fig. 6 shows a photograph of the representative water drops on the webs formed at different levels of RH. The average WCA of the PVDF webs was $81 \pm 4.6^{\circ}, 128 \pm 3.8^{\circ}, 135 \pm 3.2^{\circ}$, and $156 \pm 2.5^{\circ}$ at a RH of $2 \%, 22 \%, 42 \%$, and $62 \%$, respectively (Fig. 6a-d). The increase of the WCA as a result of the increasing $\mathrm{RH}$ is attributed to the increase in the surface roughness of the fiber webs. The cactus-like fibers exhibited the highest WCA owing to their hierarchical structures, which promotes the trapping of air between the water droplet and the surface, and prevents water from penetrating the surface of the fibers. ${ }^{55,56}$ Importantly, the electrospun PVDF cactus structure exhibited an outstanding WCA compared to the WCAs obtained from previous studies based on pristine PVDF electrospun fibers. ${ }^{57-59}$

\subsection{Effect of the RH on the piezoelectric properties of PVDF nanofiber webs}

To explore the relationship between the cactus structure and the electrical outputs of the piezoelectric nanogenerator (PENG), four PENGs based on electrospun PVDF fiber webs at different relative humidities were fabricated. For precise comparison, the PENGs were tested under the same conditions (web thickness of $100 \mu \mathrm{m}$; working area of $15 \mathrm{~cm}^{2}$; impact frequency of $5 \mathrm{~Hz}$; peak force $10 \mathrm{~N}$ ). The results showed that the voltage and current outputs of the PENGs were $0.65 \mathrm{~V}$ and $1.44 \mu \mathrm{A}$ at a $\mathrm{RH}$ of $2 \%$, $0.89 \mathrm{~V}$ and $1.87 \mu \mathrm{A}$ at a RH of $22 \%, 1.43 \mathrm{~V}$ and $2.21 \mu \mathrm{A}$ at a RH of $42 \%$, and $1.73 \mathrm{~V}$ and $2.79 \mu \mathrm{A}$ at a RH of $62 \%$ (Fig. 7 and $\mathrm{S} 3 \dagger$ ). Herein, the highest electrical outputs for the PENGs based on the PVDF cactus structure can be attributed to their extraordinary rough surface and high $F(\beta) .{ }^{44}$

\section{Conclusions}

In summary, the PVDF cactus structure was directly electrospun. The fiber webs were formed using a 22\% (DMF/ACE) PVDF solution at a solvent ratio of $1: 8$ with a voltage of 18 $\mathrm{kV}$, a tip to collector distance of $18 \mathrm{~cm}$, a feeding rate of $1.5 \mathrm{ml}$ $\mathrm{h}^{-1}$, and a $\mathrm{RH}$ of $62 \%$. The mechanism of formation for the cactus structure was explored. The results indicated that there is a negative linear relationship between the $\mathrm{RH}$ and the mechanical properties of the PVDF fiber webs, while there is a positive linear relationship between the $\mathrm{RH}$ and the physicochemical properties, as well as the piezoelectric properties of these webs. As the PVDF cactus-like nanofibers have multiple advantages represented by a high degree of roughness, interior porosity, extraordinary WCA, and good electrical outputs; this structure could serve as a potential candidate for several applications, including energy harvesting, sensors, selfcleaning surfaces, and so on.

\section{Conflicts of interests}

The authors declare that they have no competing interests.

\section{Acknowledgements}

This work is supported by the National Natural Science Foundation of China (51403033), the "Chen Guang" Project sponsored by the Shanghai Municipal Education Commission and the Shanghai Education Development Foundation (14CG34) and the Fundamental Research Funds for the Central Universities.

\section{References}

1 F. K. Ko and Y. Wan, Introduction to nanofiber materials, Cambridge University Press, Cambridge, 2014.

2 S. Jiang, G. Duan, E. Zussman, A. Greiner and S. Agarwal, ACS Appl. Mater. Interfaces, 2014, 6, 5918-5923.

3 C. Huang, H. Niu, J. Wu, Q. Ke, X. Mo and T. Lin, J. Nanomater., 2012, 2012, 7.

4 S. S. Latthe, C. Terashima, K. Nakata and A. Fujishima, Mol, 2014, 19, 4256-4283.

5 S. Jiang, S. Agarwal and A. Greiner, Angew. Chem., Int. Ed., 2017, 56, 15520-15538.

6 H. Cui, Y. Li, X. Zhao, X. Yin, J. Yu and B. Ding, Compos. Commun., 2017, 6, 63-67.

7 A. V. Stanishevsky, J. D. Wetuski and H. Yockell-Lelièvre, Ceram. Int., 2016, 42, 388-395.

8 T. Li, X. Ding, L. Tian, J. Hu, X. Yang and S. Ramakrishna, Mater. Sci. Eng., C, 2017, 74, 471-477.

9 W. Gao, L. Sun, X. Fu, Z. Lin, W. Xie, W. Zhang, F. Zhao and X. Chen, J. Mater. Chem. B, 2018, 6, 277.

10 M. Gernhardt, L. Peng, M. Burgard, S. Jiang, B. Förster, H. Schmalz and S. Agarwal, Macromol. Mater. Eng., 2018, 303, 1700248.

11 L. Peng, S. Jiang, M. Seuß, A. Fery, G. Lang, T. Scheibel and S. Agarwal, Macromol. Mater. Eng., 2016, 301, 48-55.

12 W. Liu, J. Lipner, C. H. Moran, L. Feng, X. Li, S. Thomopoulos and Y. Xia, Adv. Mater., 2015, 27, 25832588.

13 A. S. Nair, Y. Shengyuan, Z. Peining and S. Ramakrishna, Chem. Commun., 2010, 46, 7421-7423.

14 Y. Zheng, X. Gao and L. Jiang, Soft Matter, 2007, 3, 178-182. 15 A. Greiner and J. H. Wendorff, Angew. Chem., Int. Ed., 2007, 46, 5670-5703.

16 C. T. Lim, Prog. Polym. Sci., 2017, 70, 1-17.

17 N. Bhardwaj and S. C. Kundu, Biotechnol. Adv., 2010, 28, 325347.

18 S. Jiang, Y. Chen, G. Duan, C. Mei, A. Greiner and S. Agarwal, Polym. Chem., 2018, 9, 2685-2720.

19 B. Zaarour, W. Zhang, L. Zhu, X. Y. Jin and C. Huang, Text. Res. J., 2018, DOI: 10.1177/0040517518792748.

20 S. Jiang, N. Helfricht, G. Papastavrou, A. Greiner and S. Agarwal, Macromol. Rapid Commun., 2018, 39, 1700838.

21 A. Tamayol, M. Akbari, N. Annabi, A. Paul, A. Khademhosseini and D. Juncker, Biotechnol. Adv., 2013, 31, 669-687.

22 B. Bhushan and Y. C. Jung, Prog. Mater. Sci., 2011, 56, 1-108. 23 S. Priya and D. J. Inman, Energy harvesting technologies, Springer, New York, 2009. 
24 A. Podgórski, A. Bałazy and L. Gradoń, Chem. Eng. Sci., 2006, 61, 6804-6815.

25 M. Zhu, J. Han, F. Wang, W. Shao, R. Xiong, Q. Zhang, H. Pan, Y. Yang, S. K. Samal and F. Zhang, Macromol. Mater. Eng., 2017, 302, 1600353.

26 H. S. Nalwa, J. Biomed. Nanotechnol., 2014, 10, 2421-2423.

27 H. Mu, C. Li, J. Bai and W. Sun, J. Mol. Struct., 2018, 1165, 90100.

28 B. Ding, M. Wang, X. Wang, J. Yu and G. Sun, Mater. Today, 2010, 13, 16-27.

29 J. Chang, M. Dommer, C. Chang and L. Lin, Nano Energy, 2012, 1, 356-371.

30 Y. Liao, R. Wang and A. G. Fane, J. Membr. Sci., 2013, 440, 7787.

31 H. Parangusan, D. Ponnamma and M. A. A. Al-Maadeed, Sci. Rep., 2018, 8, 754.

32 C. Lang, J. Fang, H. Shao, X. Ding and T. Lin, Nat. Commun., 2016, 7, 11108.

33 P. Capkova, M. Kormunda, Z. Kolska, J. Trögl, M. Munzarova and P. Rysanek, Macromol. Mater. Eng., 2018, 303, 1700415.

34 B. Zaarour, L. Zhu, C. Huang and X. Jin, Nanoscale Res. Lett., 2018, 13, 285.

35 L. Huang, N. N. Bui, S. S. Manickam and J. R. McCutcheon, J. Polym. Sci., Part B: Polym. Phys., 2011, 49, 1734-1744.

36 S. M. Damaraju, S. Wu, M. Jaffe and T. L. Arinzeh, Biomed. Mater., 2013, 8, 045007.

37 C. Ribeiro, V. Sencadas, J. L. G. Ribelles and S. LancerosMéndez, Soft Mater., 2010, 8, 274-287.

38 M. S. S. Bafqi, R. Bagherzadeh and M. Latifi, J. Polym. Res., 2015, 22, 130.

39 H. Shao, J. Fang, H. Wang and T. Lin, RSC Adv., 2015, 5, 14345-14350.

40 C. Xing, J. Guan, Y. Li and J. Li, ACS Appl. Mater. Interfaces, 2014, 6, 4447-4457.
41 Y.-J. Kim, C. H. Ahn and M. O. Choi, Eur. Polym. J., 2010, 46, 1957-1965.

42 A. Haider, S. Haider and I.-K. Kang, Arabian J. Chem., 2015, 12, 1878-5352.

43 C. Huang, Y. Tang, X. Liu, A. Sutti, Q. Ke, X. Mo, X. Wang, Y. Morsi and T. Lin, Soft Matter, 2011, 7, 10812-10817.

44 B. Zaarour, L. Zhu, C. Huang and X. Jin, J. Appl. Polym. Sci., 2018, 136, 47049.

45 P. Lu and Y. Xia, Langmuir, 2013, 29, 7070.

46 Y.-L. Liu, Y. Li, J.-T. Xu and Z.-Q. Fan, ACS Appl. Mater. Interfaces, 2010, 2, 1759-1768.

47 H. Yu, T. Huang, M. Lu, M. Mao, Q. Zhang and H. Wang, Nanotechnol, 2013, 24, 405401.

48 M. Bachmann, W. Gordon, J. Koenig and J. Lando, J. Appl. Phys., 1979, 50, 6106-6112.

49 A. J. Lovinger, Macromol, 1982, 15, 40-44.

50 A. Salimi and A. Yousefi, Polym. Test., 2003, 22, 699-704.

51 M. Broadhurst, G. Davis, J. McKinney and R. Collins, J. Appl. Phys., 1978, 49, 4992-4997.

52 C.-T. Pan, C.-K. Yen, H.-C. Wu, L. Lin, Y.-S. Lu, J. C.-C. Huang and S.-W. Kuo, J. Mater. Chem. A, 2015, 3, 6835-6843.

53 X. Liu, S. Xu, X. Kuang, D. Tan and X. Wang, RSC Adv., 2016, 6, 109061-109066.

54 D. L. Chinaglia, R. Gregorio, J. C. Stefanello, R. A. Pisani Altafim, W. Wirges, F. Wang and R. Gerhard, J. Appl. Polym. Sci., 2010, 116, 785-791.

55 M. Ma, R. M. Hill, J. L. Lowery, S. V. Fridrikh and G. C. Rutledge, Langmuir, 2005, 21, 5549-5554.

56 L. Jiang, Y. Zhao and J. Zhai, Angew. Chem., Int. Ed., 2004, 116, 4438-4441.

57 Z. Zhou and X.-F. Wu, Mater. Lett., 2015, 160, 423-427.

58 Y. Liao, C.-H. Loh, R. Wang and A. G. Fane, ACS Appl. Mater. Interfaces, 2014, 6, 16035-16048.

59 Y. Liao, R. Wang, M. Tian, C. Qiu and A. G. Fane, J. Membr. Sci., 2013, 425, 30-39. 